\title{
Spectroscopic evaluation of QDs encapsulated with a novel biocompatible polymer for cancer diagnosis
}

\author{
Elnaz Yaghini*1, Arthur M Iga², Alexander M Seifalian² and \\ Alexander J MacRobert ${ }^{1}$
}

\begin{abstract}
Address: ${ }^{1}$ National Medical Laser Centre (NMLC), University College London, London, UK and ${ }^{2}$ Biomaterials and Tissue Engineering Centre, Division of Surgery and Interventional Science, University College London, London, UK

* Corresponding author
\end{abstract}

\author{
from Ist Scientific Meeting of the Head and Neck Optical Diagnostics Society \\ London, UK. I4 March 2009 \\ Published: 28 July 2009 \\ Head \& Neck Oncology 2009, I(SuppI I):P7 doi:I0.I I86/I758-3284-I-SI-P7
}

This abstract is available from: http://www.headandneckoncology.org/content/I/SI/P7

(c) 2009 Yaghini et al; licensee BioMed Central Ltd.

\section{Introduction}

Quantum dots (QDs) are new class of fluorescent inorganic nanocrystals which have been used for in vitro and in vivo imaging. Their unique optical properties such as broad excitation spectra, narrow emission spectrum and resistance to photobleaching make them ideal for biological labeling. Sentinel lymph node biopsy is a means of ultra-staging cancer metastasis and is now the standard of care in breast cancer surgery. Localisation of sentinel nodes is also important in the treatment of head and neck cancer. Current tracers for SLN biopsy include the blue dye have various limitations that could be overcome by quantum dots that emit in near infrared range $(>700 \mathrm{~nm})$. To safely deliver QDs they must be encapsulated in a biocompatible coating. In this study we encapsulate CdTe QDs with new nanocomposite material based on a silsesquioxane modified poly (carbonate-urea) urethane polymer, and evaluated their spectroscopic properties.

\section{Aim}

Developing new biocompatible QDs and investigating their spectroscopic properties versus that of un-coated one.

\section{Materials and methods}

QDs solution was irradiated with $670 \mathrm{~nm}$ laser diode (Hamamatsu LD 4000) to examine the photostability of the QDs, using CCD spectrometer. Fluorescence lifetime was measured using time-correlated single photon counting method.

\section{Results}

Encapsulation with the polymer enhances the aqueous solubility and biocompatibility of the nanocrystals, and provides an opportunity to modify the surface for biomedical applications. QDs are relatively resistance to photobleaching, the presence of the polymer coating did not appear to significantly modify the photobleaching threshold and the fluorescence lifetime of the quantum dot. 